\title{
Supporting Information: The Origin of Apparent Slow Solvent Dynamics in Concentrated Polymer Solutions
}

\author{
Till Böhmer*,a, Robin Horstmann ${ }^{\mathrm{a}}$, Jan Philipp Gabriel ${ }^{\mathrm{b}}$, Florian Pabst ${ }^{\mathrm{a}}$, Michael Vogel ${ }^{\mathrm{a}}$, and \\ Thomas Blochowicz ${ }^{\mathrm{a}}$ \\ anstitute for Condensed Matter Physics, Technical University of Darmstadt, 64289 \\ Darmstadt, Germany

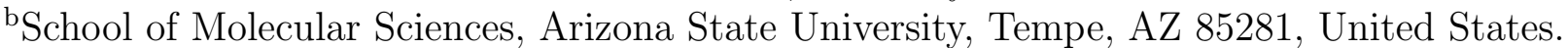 \\ *Email: tboehmer@fkp.tu-darmstadt.de
}

October 16, 2021

\section{Parametrization of the PMMA Repeat Unit}

The parametrization of PMMA was taken from the web service "automated topology builder" (ATB), see the united atom model for molid 367617 and hash $572 \mathrm{dd}$. The full polymer chain with 50 repeat units is too large for parametrization with more sophisticated methods. However, such detailed parametrization is irrelevant for our purpose. To produce polymer chains of arbitrary length, we simplified the model to a single charge neutral repeat unit, see Figure SI. 1. Thus, the partial charges had to be adjusted, presented in Table SI. 1, and deviate slightly from the original ATB parametrization. The parametrization of bonds, bond angles and dihedrals are taken from the parametrization of the short polymer chain. In particular, repeat units are linked using all three bonded potentials.

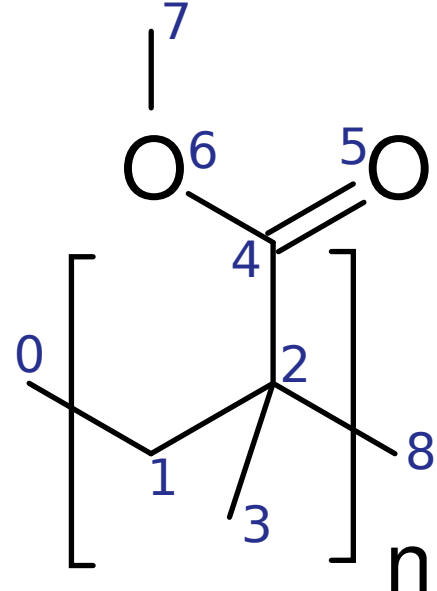

Figure SI. 1: (a) Chemical formula of the united atom representation of the repeat unit of PMMA. All hydrogen atoms are merged with their respective carbon atoms.

\begin{tabular}{llc} 
Index & atomtype & q in e \\
\hline 1 & CH2 & 0.069 \\
2 & C & -0.021 \\
3 & CH3 & 0.070 \\
4 & CPos & 0.305 \\
5 & OEOpt & -0.358 \\
6 & OE & -0.275 \\
7 & CH3 & 0.210
\end{tabular}

Table SI. 1: (b) Atomtype in the ATB GROMOS forcefield and partial charge $q$ of the atoms of PMMA repeat units. The index is the blue number in (a). 


\section{Combination of DDLS Techniques}

In this section we discuss details of the combination of DDLS data from photon correlation spectroscopy (PCS) and Tandem Fabry-Perot interferometry (TFPI). Data from both techniques measured at the same temperatures are analyzed in $\chi^{\prime \prime}$ representation, obtained following the procedure explained in the experimental section of the main publication and shown in Figure SI. 2 (a) and (b). Data combination is based on the fact that both techniques resolve different aspects of the solvent structural relaxation process at most temperatures. The process can be described by the Havriliak-Negami (HN) equation ${ }^{1}$

$$
\chi_{\alpha}^{\prime \prime}=\operatorname{Im}\left(\frac{\Delta \chi}{\left(1+(i \omega \tau)^{a_{\mathrm{HN}}}\right)^{b_{\mathrm{HN}}}}\right),
$$

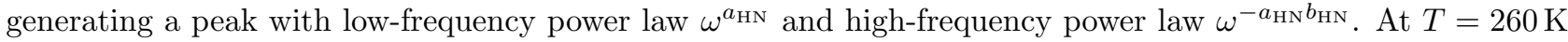
and above, the curve fitting is entirely based of the TFPI data, as no signature of the solvent structural relaxation process is observed in PCS. At lower temperatures the process is fit in TFPI, with the value of $a_{\mathrm{HN}}$ being confirmed from fitting the onset of the structural relaxation process in PCS by a power law, as indicated in panel (b) of the figure. At the lowest temperature, no information regarding $\omega^{a \mathrm{HN}}$ can be obtained from the TFPI data, thus its determination is fully based on the PCS data. Due to the increase of dynamic heterogeneity in the mixture with decreasing temperature, the slope parameter $a_{\mathrm{HN}}$ steadily decreases during cooling (see the inset of panel (a)).

After curve fitting the HN equation to the TFPI data, with the value of $a_{\mathrm{HN}}$ corresponding to the low-frequency flank in the PCS data, the latter are shifted in intensity to match the HN power laws of both data sets in absolute values for each temperature. This procedure is sketched in Figure SI. 2 (c) at a moderate temperature. Finally, the data sets are joined, resulting in a set of broadband DDLS data, with the relaxation intensities being comparable between data at different temperatures. 

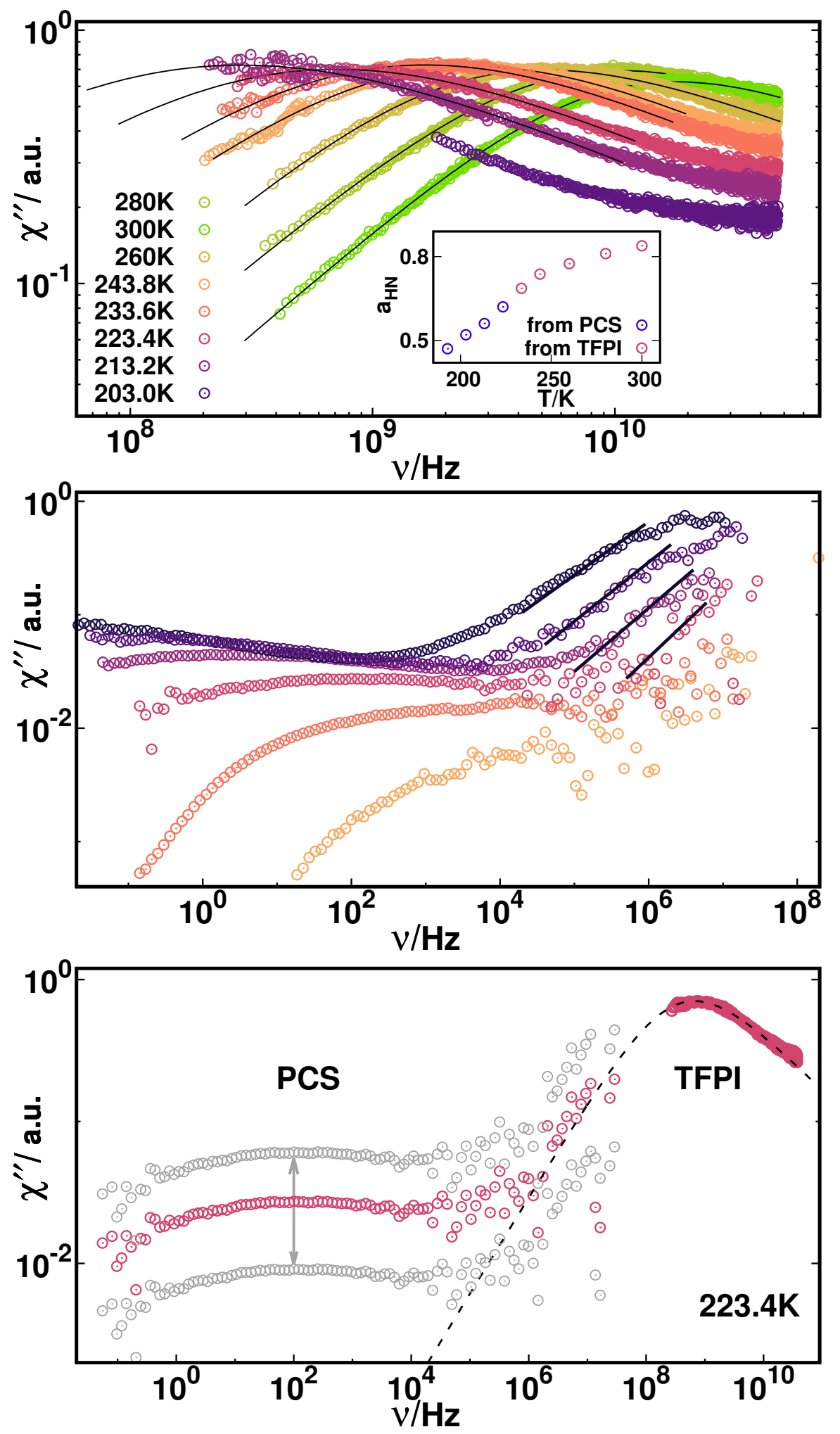

Figure SI. 2: (a) TFPI data with HN fits according to Eq. (1). The temperature dependence of the slope parameter $a_{\mathrm{HN}}$ is shown in the inset. Temperatures where $a_{\mathrm{HN}}$ was estimated from PCS or TFPI are shown as a blue or red symbol, respectively. Higher temperatures than in Figure 2 of the main publication are also included. (b) PCS data at the same temperatures as shown in Figure 2 of the main publication. The lowfrequency power law of the solvent structural relaxation process is estimated from a linear fit at the lowest four temperatures, shown as black lines. (c) Schematic illustration of the procedure to combine TFPI and PCS data sets by shifting the PCS data in intensity until they agree with the power law fit of the low-frequency flank of the TFPI data. 


\section{Dependence on Legendre Polynomial and Molecular Vector}

In the main text we present MD simulation results of correlation functions calculated for the normalized molecular dipole moment vectors and the first order Legendre polynomial (LP), mimicking the situation in a BDS experiment. In DDLS experiments the reorientation of the principal axis of the molecular optical anisotropy tensor is probed via the second order LP. Therefore, it is useful to determine the dependence of the cross-correlations on the considered molecular vector and the rank $\ell$ of the LP. By this we aim to justify our qualitative comparison of DDLS data and the MD simulation data shown in the main publication.

We use two distinct vectors in the frame of the 2-picoline molecule, the dipole moment $\vec{\mu}$ and the normal vector $\vec{n}$ of the plane defined by the ring. For each of the vectors we calculate the $\ell=1$ and $\ell=2$ coherent rotational correlation functions $C_{1}$ and $C_{2}$ as well a their incoherent and cross-correlation contributions. The correlation functions $C_{1, \vec{\mu}}$ and $C_{2, \vec{n}}$ are observed in BDS and DDLS, respectively, while the other two combinations are only accessible in the simulation. In Figure SI. 3(a) the normalized cross-correlation contribution of the solvent is shown for the different choices of $\ell$ and molecular vector. For $\vec{\mu}$, both LPs produce qualitatively the same correlation function. However, because the angular dependence is stronger for $C_{2}$ than for $C_{1}$, the crosscorrelations are weaker for the former than the latter. Because of this difference, we focus on $\ell=1$ correlation functions in the main text. The cross-correlations of $C_{2, \vec{n}}$ are slightly stronger than those of $C_{2, \vec{\mu}}$. The increase in cross-correlations of $C_{2, \vec{n}}$ from $10^{-9} \mathrm{~s}$ to $10^{-7} \mathrm{~s}$ is caused by a redistribution of the coherent correlation. $C_{\text {cross }}$ may increase when $C_{\text {coh }}$ decays slower than $C_{\text {inc }}$, which is the case here. Because cross-correlations in $C_{2, \vec{n}}$ are weaker between neighboring 2-picoline molecules than for distinct replacement, they increase at intermediate times shorter than the relaxation of the matrix. Thus, cross-correlations are present in both, first and second order LPs and also appear in $C_{2, \vec{n}}$ probed in DDLS. Therefore, a comparison of $C_{1, \vec{\mu}}$ from MD simulation and DDLS data probing $C_{2, \vec{n}}$ is reasonable.

Additionally, we show the temperature dependent correlation functions $C_{\mathrm{coh}}, C_{\text {inc }}$ and $C_{\text {cross }}$ calculated for $\ell=2$ and the normal vector $\vec{n}$ in Figure SI. 3(b-d). For better comparison we use the same presentation as in Fig. 3 of the main publication. Compared to $C_{1, \vec{\mu}}$, the temperature dependence of the long-time crosscorrelations is stronger in $C_{2, \vec{n}}$. This apparent discrepancy is a mere effect of the higher angular sensitivity of the LP $P_{\ell=2}$, which also leads to a stronger temperature dependence of the cross-correlation intensity. 

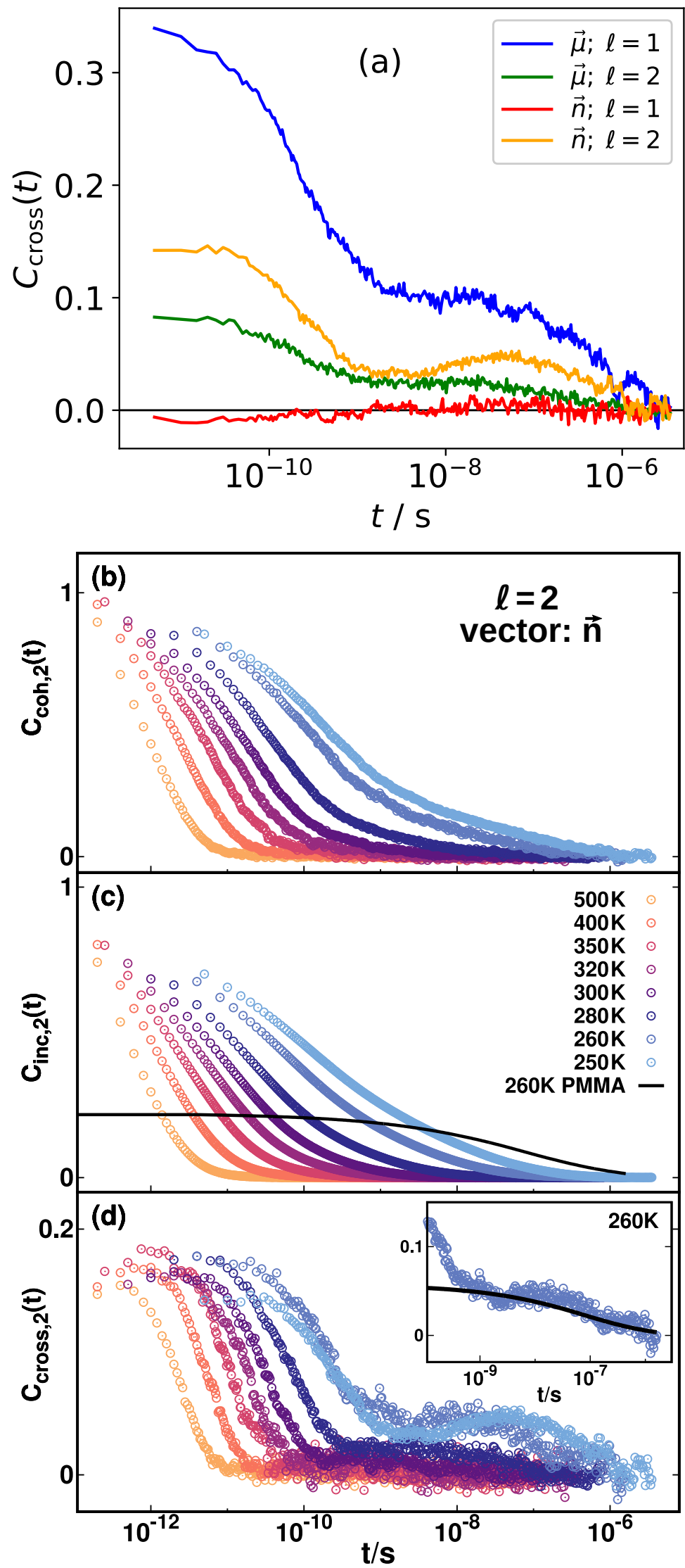

Figure SI. 3: (a) Cross-correlations of 2-Picoline molecules at $250 \mathrm{~K}$ calculated using the vectors $\vec{\mu}$ and $\vec{n}$ and Legendre polynomials of the ranks $\ell=1$ and $\ell=2$, respectively. (b)-(d) Same presentation as in Fig. 3 of the main publication, but with correlation functions calculated for $\vec{n}$ and $\ell=2$ to mimic the situation in a DDS experiment. 


\section{Correlation between slow cross-correlation decay and PMMA relaxation}

In the inset of Fig. 3(c) in the main publication we present a correlation between the slow cross-correlation decay and the PMMA segmental relaxation regarding relaxation time. In Figure SI. 4 we present the temperature dependence of this correlation. Here, the PMMA relaxation curves were scaled to correspond to the plateau height of the cross-correlations. As can be seen, the correlation holds well for all investigated temperatures.

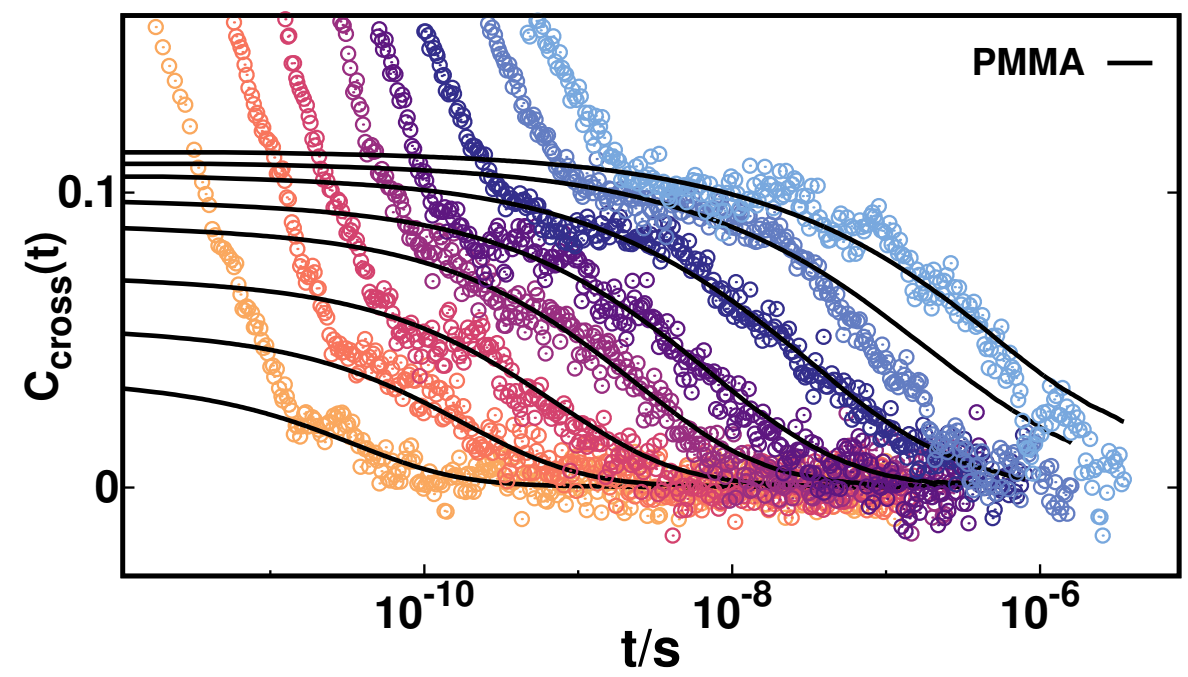

Figure SI. 4: Comparison of the slow decay of the solvent cross-correlations (symbols) and the PMMA segmental relaxation(black lines) similar to the example shown in Fig. 3(c) in the main publication. The colors chosen for the different temperatures correspond to the ones in Figure SI. 3(b-d).

\section{MD Simulation Snapshots of the Replacement Process}

In Figure SI. 5 we show a part of the simulated system at two times along a trajectory to substantiate the replacement process sketched in Fig. 5 of the main text. Two solvent molecules replacing each other are colored red and blue, respectively. In the time interval between the two snapshots, the red molecule has replaced the blue one at a preferred location close to the polymer matrix. Their respective orientations in this location coincide. During this replacement process, no significant polymer relaxation has taken place.
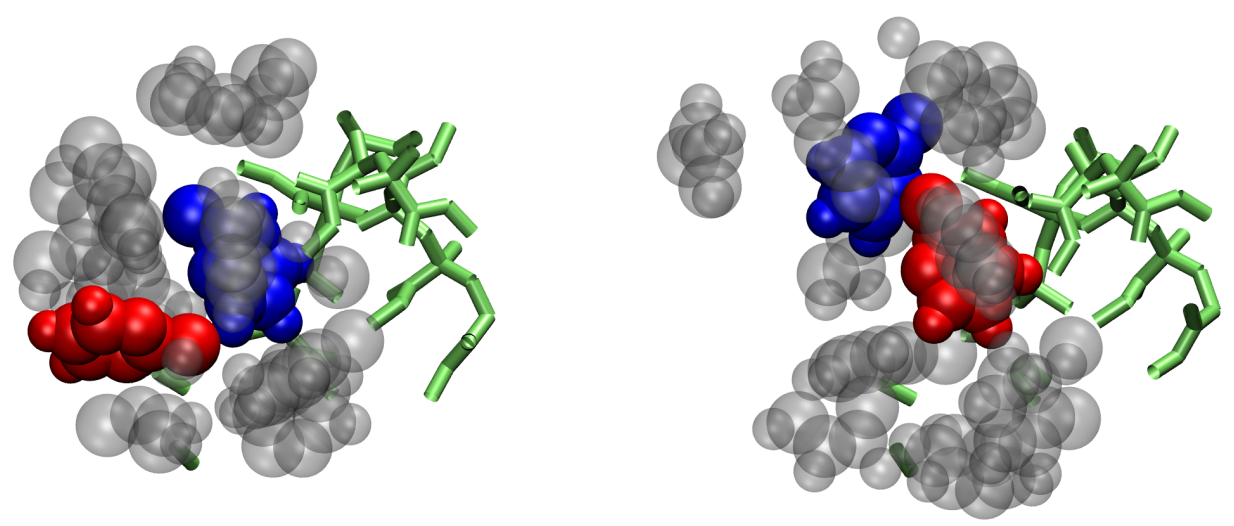

Figure SI. 5: Two snapshots of the same part of the simulated system at different times. The time interval between the snapshots amounts to $670 \mathrm{ps}$. The atoms of the solvent molecules involved in the replacement process are shown as red and blue spheres, respectively, while those of other solvent molecules are colored grey. The polymer matrix is represented by green tubes. 


\section{Density Correlation Function}

In mode-coupling theory $(\mathrm{MCT}),{ }^{2}$ single-particle and collective density correlation functions have been shown to differ for asymmetric binary mixtures ${ }^{3}$ To relate this MCT finding to the cross-correlation mechanism derived based on reorientation dynamics in the main text, we calculate also density correlation data from our MD simulations.

Figure SI. 6 displays the incoherent scattering functions $S_{\text {inc }}(q, t)$ and the coherent scattering functions $S_{\text {coh }}(q, t)$ calculated from the center-of-mass positions of the 2-picoline molecules of the investigated binary mixture for various absolute values of the scattering vector $q$ and at $250 \mathrm{~K}$. As expected, the decays of both functions shift to longer times with increasing length scale (or decreasing $q$ ). Like $C_{\text {coh }}$ in the main publication,

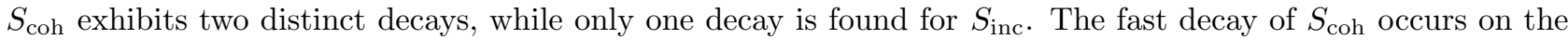
same time scale as that of $S_{\text {inc }}$, whereas the slower decay of $S_{\text {coh }}$ is clearly separated. Consequently, no slow species is found in $S_{\text {inc }}$, while the coherent results agree at least qualitatively with the theoretical expectation from MCT and provide evidence for a slow process. The finding of cross-correlation contributions on the time scale of the segmental relaxation of the polymer in both orientational and density correlations implies a direct relation of both phenomena.
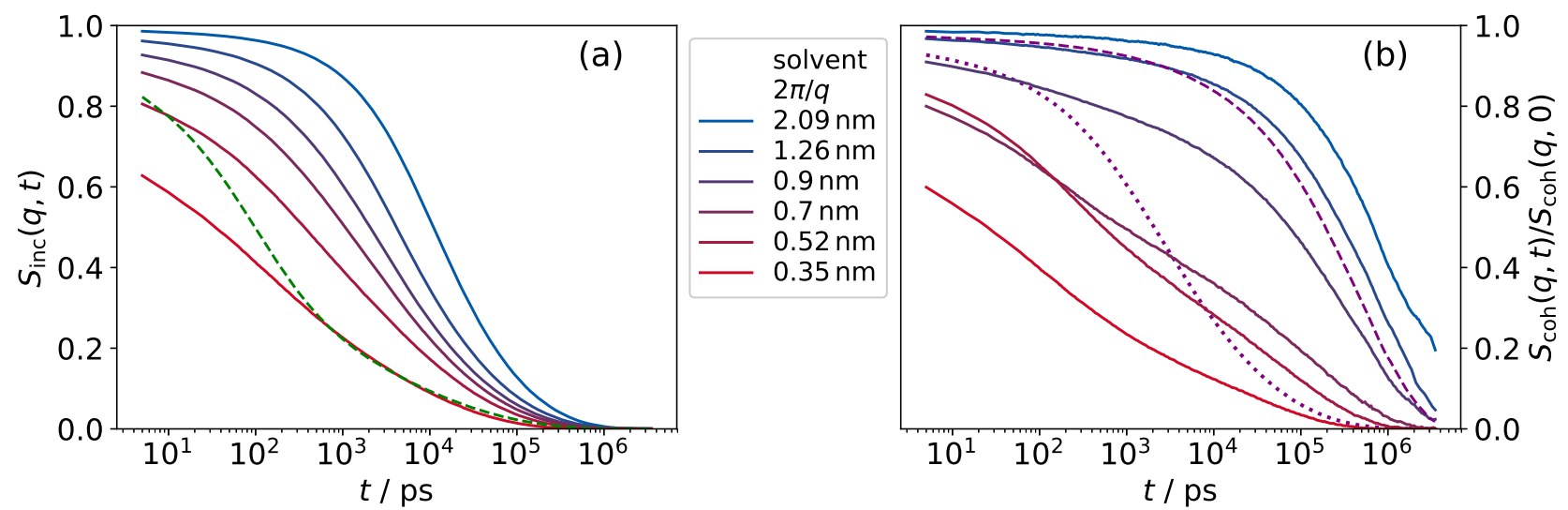

Figure SI. 6: Simulated scattering functions calculated from the center-of-mass positions of the 2-Picoline molecules for various absolute values of the scattering vector $q$ and at $250 \mathrm{~K}$ : (a) incoherent scattering function $S_{\text {inc }}(q, t)$ and (b) coherent scattering function $S_{\text {coh }}(q, t)$. The respective length scales $2 \pi / q$ are given in the legend. In panel (a), the rotational correlation function $C_{\text {inc }}(t)$ from the main text is included as green dashed line. In panel (b), the dotted and dashed purple lines are $S_{\text {inc }}(q, t)$ for the 2-Picoline and the PMMA segments, respectively, both for $2 \pi / q=0.9 \mathrm{~nm}$ and $250 \mathrm{~K}$. 


\section{Solvent Cross-Correlations in an Aqueous $\varepsilon$-Polylysine Solution}

To substantiate our argument in the main text that the cross-correlation mechanism is not limited to the studied 2-picoline/PMMA mixture but applies also to other solvent-solute systems, we present first MD simulation results for an aqueous $\varepsilon$-polylysine (PLL) solution in this section. The simulated system comprises $12 \varepsilon$-PLL chains with 32 repeat units and 5543 water molecules, corresponding to a water concentration of $67 \mathrm{wt} \%$. We used the AMBER based force field ff03ws ${ }^{4}$ together with the TIP4P/2005 model of water. ${ }^{5}$ Missing parameters for bonded interactions were adopted from the general AMBER force field and partial charges were adjusted with restrained electrostatic potential (RESP) fitting. ${ }^{6,7}$ The simulated system was cubic with a box length of $6.1449 \mathrm{~nm}$.

The simulations were performed using the GROMACS 2018 simulation package. ${ }^{8,9}$ The leapfrog integrator with a time step of $2 \mathrm{fs}$ was used and periodic boundary conditions were employed. Simulations to equilibrate the density were performed in the isothermal-isobaric (NPT) ensemble, applying the velocity-rescaling thermostat ${ }^{10}$ and Berendsen barostat ${ }^{11}$ to set the temperature $T=300 \mathrm{~K}$ and the pressure $P=1$ bar. Production runs were performed in the canonical (NVT) ensemble. Water and non-water atoms were coupled to separate heat baths. The LINCS algortithm ${ }^{12}$ was used to constrain all bonds. Non-bonded pair interactions were calculated up to a cutoff of $1.4 \mathrm{~nm}$ beyond which the Particle-Mesh Ewald method ${ }^{13}$ with a Fourier spacing of $0.12 \mathrm{~nm}^{-1}$ was applied to consider long-range Coulomb interactions and dispersion forces. During the equilibration, the cutoff was $1.2 \mathrm{~nm}$. The lengths of the NPT equilibration run and NVT production run were $10 \mathrm{~ns}$ and $200 \mathrm{~ns}$, respectively.

Figure SI. 7(a) shows the rotational correlation functions $C_{\mathrm{coh}}(t), C_{\mathrm{inc}}(t)$, and $C_{\mathrm{cross}}(t)$ for the dipole moment vector of the water molecules at $300 \mathrm{~K}$ and the $\ell=1 \mathrm{LP}$. We see that $C_{\mathrm{coh}}(t)$ has a long-time tail, which is absent in $C_{\text {inc }}(t)$ and results from cross-correlations $C_{\text {cross }}(t)$. A comparison with $C_{\text {inc }}(t)$ for the dipole moments of the lysine segments reveals that the long-time tail decays on the timescale of the peptide reorientation. This is confirmed by the corresponding susceptibilities in Figure SI. 7(b). One may expect that the long-time tail evolves into a separate slow process when the time scales of water and peptide dynamics further separate upon cooling. Thus, the cross-correlation effect is not limited to the 2-picoline/PMMA mixture studied in the main text, but rather occurs also for the water/ $\varepsilon$-PLL solution. Therefore, we anticipate that the proposed cross-correlation mechanism applies to various kinds of dynamically asymmetric binary mixtures.
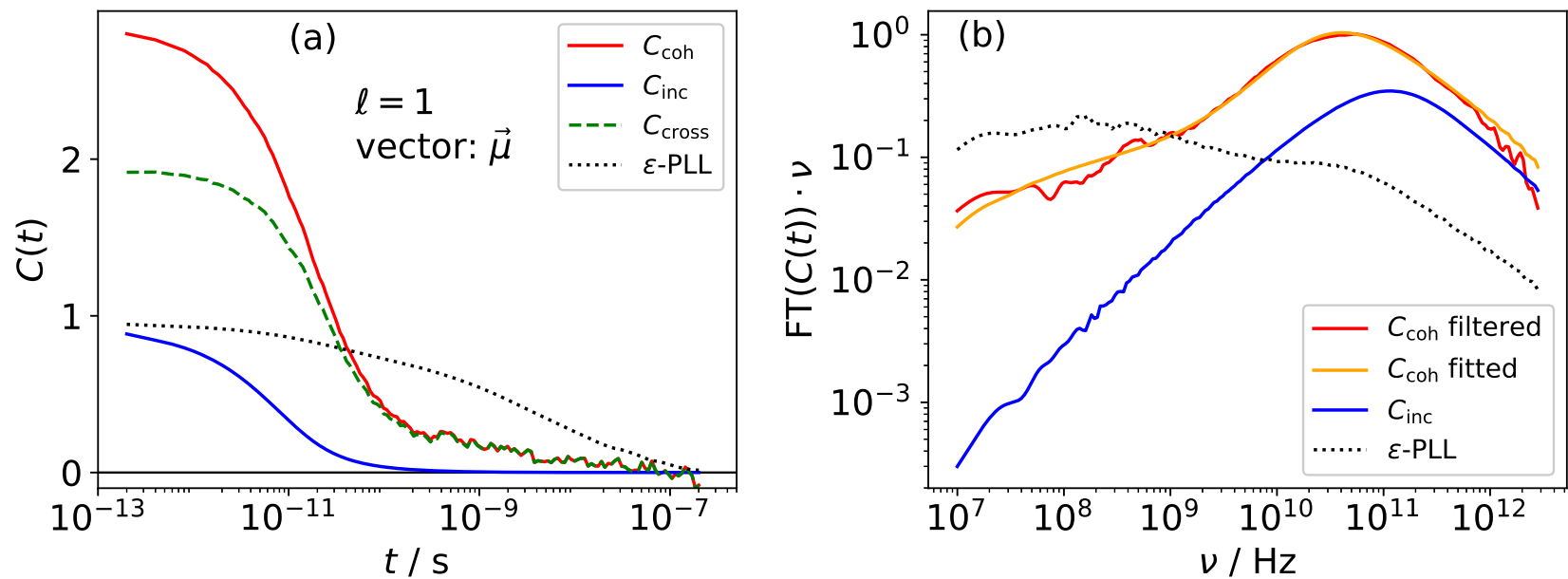

Figure SI. 7: Different measures of water dynamics obtained from MD simulations of an aqueous $\varepsilon$-PLL solution at $300 \mathrm{~K}$ : (a) Rotational correlation functions $C_{\text {coh }}(t), C_{\text {inc }}(t)$, and $C_{\text {cross }}(t)$ calculated from the time-dependent dipole moment vectors of water for $\ell=1$ according to Eqs. (1)-(3) of the main text. For comparison, the incoherent correlation function obtained from dipole moments of the individual lysine segments for $\ell=1$ is included as black dotted line. (b) Dynamic susceptibilities corresponding to the various correlation functions in panel (a). Exploiting the fluctuation-dissipation theorem, they were computed by multiplying the Fourier transform of the correlation functions by the frequency $\nu$. To improve the visibility of the slow process, we show also the results obtained from an appropriate fit of $C_{\mathrm{coh}}(t)$. 


\section{References}

[1] Havriliak, S.; Negami, S. A complex plane representation of dielectric and mechanical relaxation processes in some polymers. Polymer 1967, 8, 161-210.

[2] Gotze, W.; Sjogren, L. Relaxation processes in supercooled liquids. Rep. Prog. Phys. 1992, 55, 241-376.

[3] Bosse, J.; Kaneko, Y. Motion of interacting particles in a disordered medium. Prog. Theor. Phys. Supp. 1997, 126, 13-20.

[4] Best, R. B.; Zheng, W.; Mittal, J. Balanced Protein-Water Interactions Improve Properties of Disordered Proteins and Non-Specific Protein Association. J. Chem. Theory Comput. 2014, 10, 5113-5124.

[5] Abascal, J.; Vega, C. A general purpose model for the condensed phases of water: TIP4P/2005. J. Chem. Phys. 2005, 123, 234505.

[6] Wang, J.; Wolf, R.; Caldwell, J.; Kollman, P.; Case, D. Development and testing of a general amber force field. J. Comput. Chem. 2004, 25, 1157-1174.

[7] Bayly, C.; Cieplak, P.; Cornell, W.; Kollman, P. A well-behaved electrostatic potential based method using charge restraints for deriving atomic charges: the RESP model. J. Phys. Chem. 1993, 97, 10269-10280.

[8] Van Der Spoel, D.; Lindahl, E.; Hess, B.; Groenhof, G.; Mark, A.; Berendsen, H. GROMACS: fast, flexible, and free. J. Comput. Chem. 2005, 26, 1701-1718.

[9] Hess, B.; Kutzner, C.; van der Spoel, D.; Lindahl, E. GROMACS 4: algorithms for highly efficient, loadbalanced, and scalable molecular simulation. J. Chem. Theory Comput. 2008, 4, 435-447.

[10] Bussi, D., Gand Donadio; Parrinello, M. Canonical sampling through velocity rescaling. J. Chem. Phys. 2007, 126, 014101.

[11] Berendsen, H.; Postma, J. v.; van Gunsteren, W.; DiNola, A.; Haak, J. Molecular dynamics with coupling to an external bath. J. Chem. Phys. 1984, 81, 3684-3690.

[12] Hess, B.; Bekker, H.; Berendsen, H.; Fraaije, J. LINCS: a linear constraint solver for molecular simulations. J. Comput. Chem. 1997, 18, 1463-1472.

[13] Darden, T.; York, D.; Pederson, L. Particle mesh Ewald: An N.log(N) method for Ewald sums in large systems. J. Chem. Phys. 1993, 98, 10089. 